Ann. Sci. For., 1984, 41 (2), 171-194

\title{
Relations entre les composantes densitométriques et l'état de surface de planches rabotées chez Pseudotsuga menziesii
}

\author{
G. NEPVEU et T. TRAN NGOC * \\ I.N.R.A.. Station de Recherches sur la Qualité des Bois \\ Centre de Recherches forestieres de Nancy \\ Champenoux, F 54280 Seichamps \\ * Chaire de Technologie du Bois \\ Ecole supérieure de Sylviculture de Quang Ninh, Viet Nam
}

\section{Résumé}

Trois critères ont été détcrminés sur les courbes d'état de surface de planches rabotées provenant de 8 jeunes douglas (Pseudotsuga menziesii). On a étudié ensuite les relations entre ces critères et les composantcs densitométriques obtenues par radiographie aux rayons $X$, en distinguant la variabilité intraarbre (entre cernes) et la variabilité individuelle (entre arbres).

Les principatix résultats sont les suivants :

- le bois juvénile présente des critères de rugosité particuliers qui peuvent siexpliquer par les composantes densitométriques spéciales de ce bois;

- il existe unc variabilité individuelle significative pour la qualité des états de surface des planches rabotées;

- l'état de surface est lié, aux niveaux intra et interindividuel, aux composantes densitométriques;

- parmi ces composantes densitométriques, la densité minimum de cerne est un critc̀re déterminant pour expliquer la rugosité; on estime qu'un gain de $65 \mathrm{~kg} / \mathrm{m}^{3}$ pour la densité minimum peut améliorer la rugosité de 40 p. 100.

\section{Introduction}

L'état de surface est un des critères essentiels servant à définir la qualité dans les industries du bois, notamment du fait qu'il conditionne les pertes de matière au rabotage et au ponçage. Les éléments qui contribuent à donner un bon état de surface se séparent en deux grands groupes : les premiers proviennent des procédés industriels, les seconds tiennent aux propriétés intrinsèques du bois, à savoir des défauts tels que nouds, contrefil, .... ou bien à la nature du matériau (densité, ...) en l'absence de toute anomalic du plan ligneux.

Sćparer l’influence de ces facteurs pour apprécier leur poids respectif est particulièrement important, tout spécialement pour l'améliorateur forestier soucieux de savoir si la sélection à l'intérieur d’une espèce de génotypes à plans ligneux particu- 
liers aura pour conséquence, sur le plan inclustriel, une meilleure qualité des produits finis.

Les éludes consacrécs à cette question sont rares: nous signalerons celle de Keller \& PERrin (1980) qui ont procédé au déroulage en simulation de quelques conifères et ont constaté que la diminution d'hétérogénéité du bois (différence entre densités maximum el minimum du cerne) en changeant d'espèce conduisait à une amélioration des états de surface.

Le but du présent travail a été d'observer les relations entre les variations de la densité du bois intraabre et interarbre el quelques critères décrivant les états de surface des planches rabotées qui en proviennent.

\section{Matériel et méthodes}

L'étude a porté sur 8 jeunes douglas (Pseudotsuga menziesii) présentant entre eux, au vu de mesures préalables, des variations sensibles d'hétérogénéité du bois, ce paramètre, a priori important, étant mestué pour chaque arbre par la moyenne portant sur un certain nombre de cernes des différences entre densités maximum et minimum de cernes.

Cette essence a été choisic car elle fait l'objet à l'heure actuelle en France d'un programne d’amélioration génétique de grande ampleur (études de variabilité, sélection, vergers à graines) se proposant de prendre en considération la qualité intrinsèque du bois. Il est donc de première importance de signaler aux généticiens les critères de densité à sélectionner, ectle décision étant liée à l'impact de ces critères sur laptitude technologique du bois en question.

\subsection{Confection des éprouvettes et mestres}

Dans la bille de pied de chaque arbre. on a découpé une planchette passant par laxe de larbre dont les cotes étaient les suivantes:

- $300 \mathrm{~mm}$ de longueur :

- $160 \mathrm{~mm}$ de largeur ;

- $\quad 5 \mathrm{~mm}( \pm 0.1 \mathrm{~mm})$ d'épaisseur après rabotage.

Le rabotage a été effectué de la manière suivante :

- larbre porte-outil a deux couteaux; sa vitesse de rotation est d'environ 4500 tours par minule:

- une face a été dégauchic avec une avance "manuelle";

- la seconde face (celle qui sera éludice) a été rabotée avec une avance de 4,2 mètres par minute.

L'humidité des 8 planches-échantillons a été contrôlée ; elle est comprise entre 10 et 11 p. 100 . La direction d'avance est naturellement parallèle à l'axe des planches. le rabotage commençant toujours par la partie supérieure de la planche. 

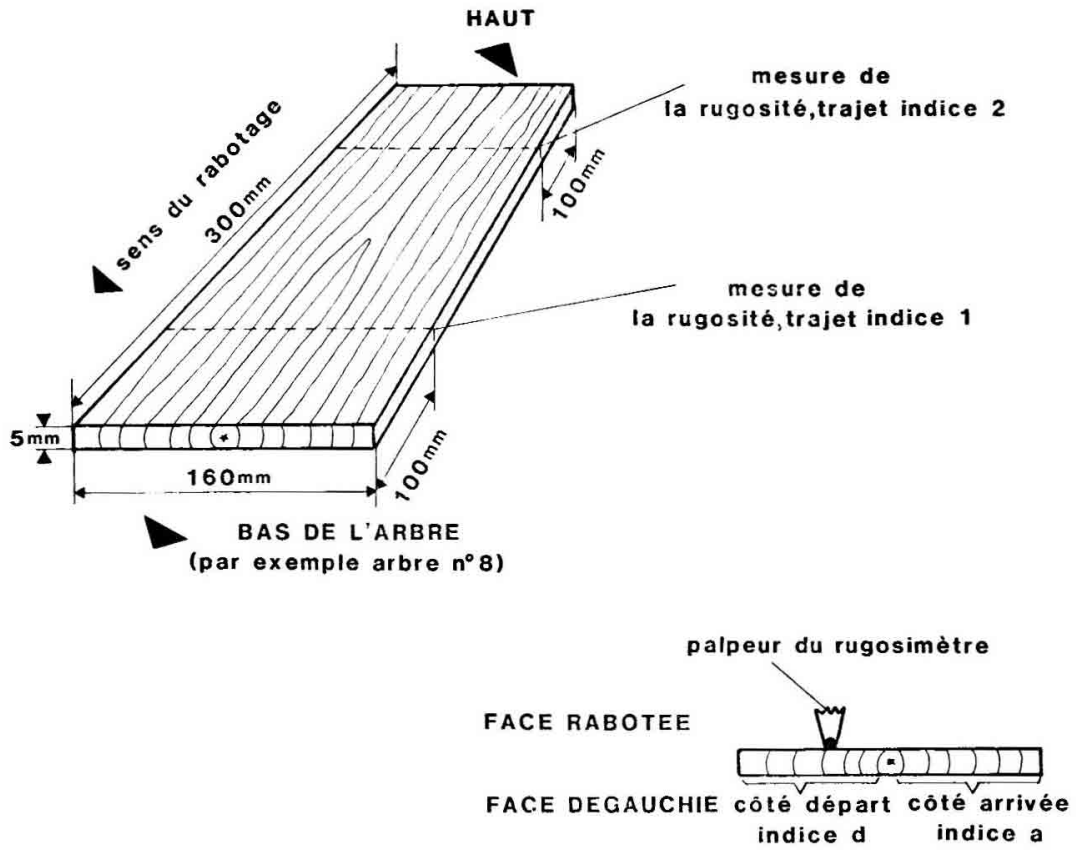

déplacement de la planche sur le tour

découpage d'un parallélipipède de $5 \mathrm{~mm}$ de large

$(2,5 \mathrm{~mm}$ de part et dautre du trajet du rugosimètre $)$

\section{COTE DE LA FACE \\ RABOTEE}

zone explorée

au microdensitomètre

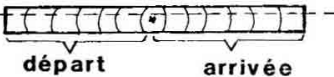

du rugosimétre

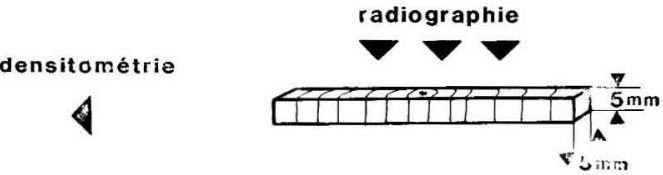

FIG. 1

Obtention des éprouvettes et mesures.

Samples obtainments and measures.

- Sens du rabotage - Planing direction.

- Mesure de la rugositć - Smoothness measure.

- Trajet - Passage.

- Bas de l'arbre - Tree base.

- Face rabotée - Planed side.

- Face dégauchie - Surfaced side.

- Côté départ - Starting side.

- Côté arrivée - Arrival side.

- Palpeur du rugosimètre - Stylus of smoothness apparatus.

- Déplacement de la planche sur le tour - Board displacement on the lathe.

- Découpage d'un parallélipipède de $5 \mathrm{~mm}$ de large $(2,5 \mathrm{~mm}$ de part et d'autre du rugosimètre) - Sawing of a parallelipiped $5 \mathrm{~mm}$ wide $(2,5 \mathrm{~mm}$ on both sides of smoothness apparatus passage).

- Zone explorée au microdensitomètre - Zone scanned by microdensitometer. 
Ensuite, les élats de surface des planches ont été mesurés sur la face rabotée. ceci suivant deux tracés parallèles perpendiculaires au fil du bois sur chaque planche (fig. 1). On a tutilisé pour ce faire un rugosimètre spécialement mis au point à la Station de Recherches sur la Qualité des Bois (Keltel et al., 1980). L'échantillon. fixé sur un tour, défile devant un stylet terminé par une extrémité hémisphérique de $1 \mathrm{~mm}$ de rayon qui, parcourant une ligne droite sur la surface de la planche (en l'occurrence perpendiculaire au fil du bois) suit les irrégularités de cette surface et transmet ses déplacements à un capteur. Lès variations détectées par le stylet (dépressions et pics) sont transcrites, agrandies. sur un graphique.

Après oblention des deux courbes d'états de surface, on a découpé deux parallélépipèdes de bois diamétraux de $5 \mathrm{~mm}$ de large dont les axes représentent les deux trajets du rugosimètre sur chacune des planches. Ceci a été réalisé en utilisant des scies-fraises jumelées (Thilrcitin \& Plirkin. 1972). Ces deux parallélépipèdes ont ensuite été radiographiés dans le sens axial et on a obtenu, par microdensitométrie. les courbes de variation de densité du bois en continu du cour vers l'écorce (Polge. 1966): l'exploration du lilm s'est effectuéc an voisinage de la face sur laquelle l'état de surface al élé mesuré.

\subsection{Variables prises en considération}

La figure 2 présente, pour l'individu 8, trajet 1, la courbe d'état de surface (côtés " départ " et "arrivée »), la courbe densitométrique correspondante, ainsi que le positil de la radiographie.

ARBRE 8 Traet 1
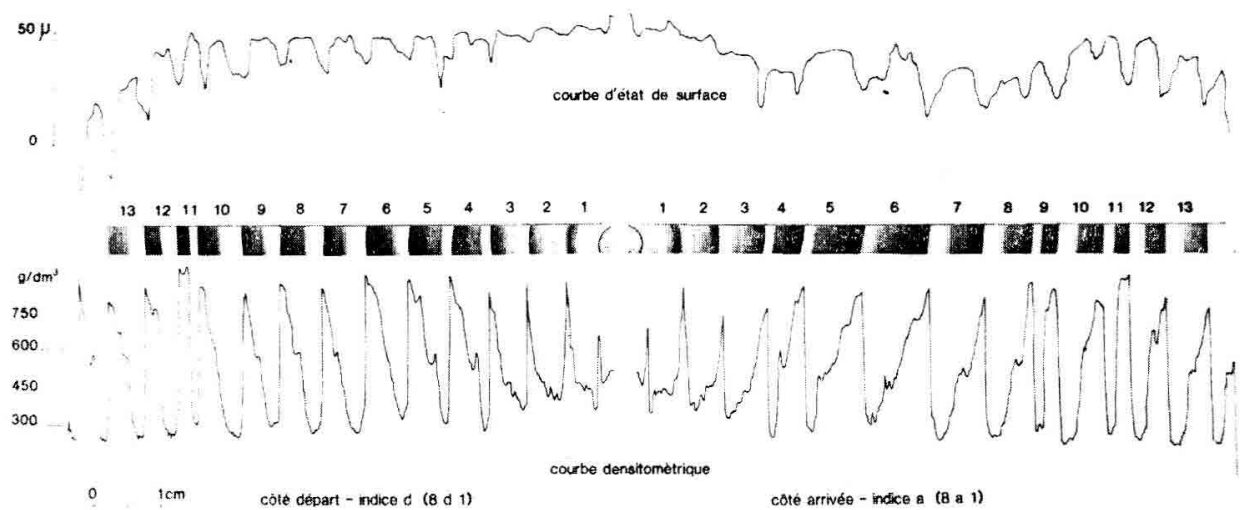

FIG. 2

Courbe d'état de surface, positif de radiographie et courbe densitométrique pour l'arbre 8 , trajet 1, côtés arrivée et départ.

Smoothness curve, X-ray radiography positive and densitometric curve for tree No. 8 , passage No. 1, arrival and starting sides.

- Courbe d'état de surface - Smoothness curve.

- Courbe densitométrique - Densitometric curve. 
Critères relevés sur les courbes d'état de surface $\left\{\begin{array}{l}h \\ a / b \\ S / b\end{array}\right.$

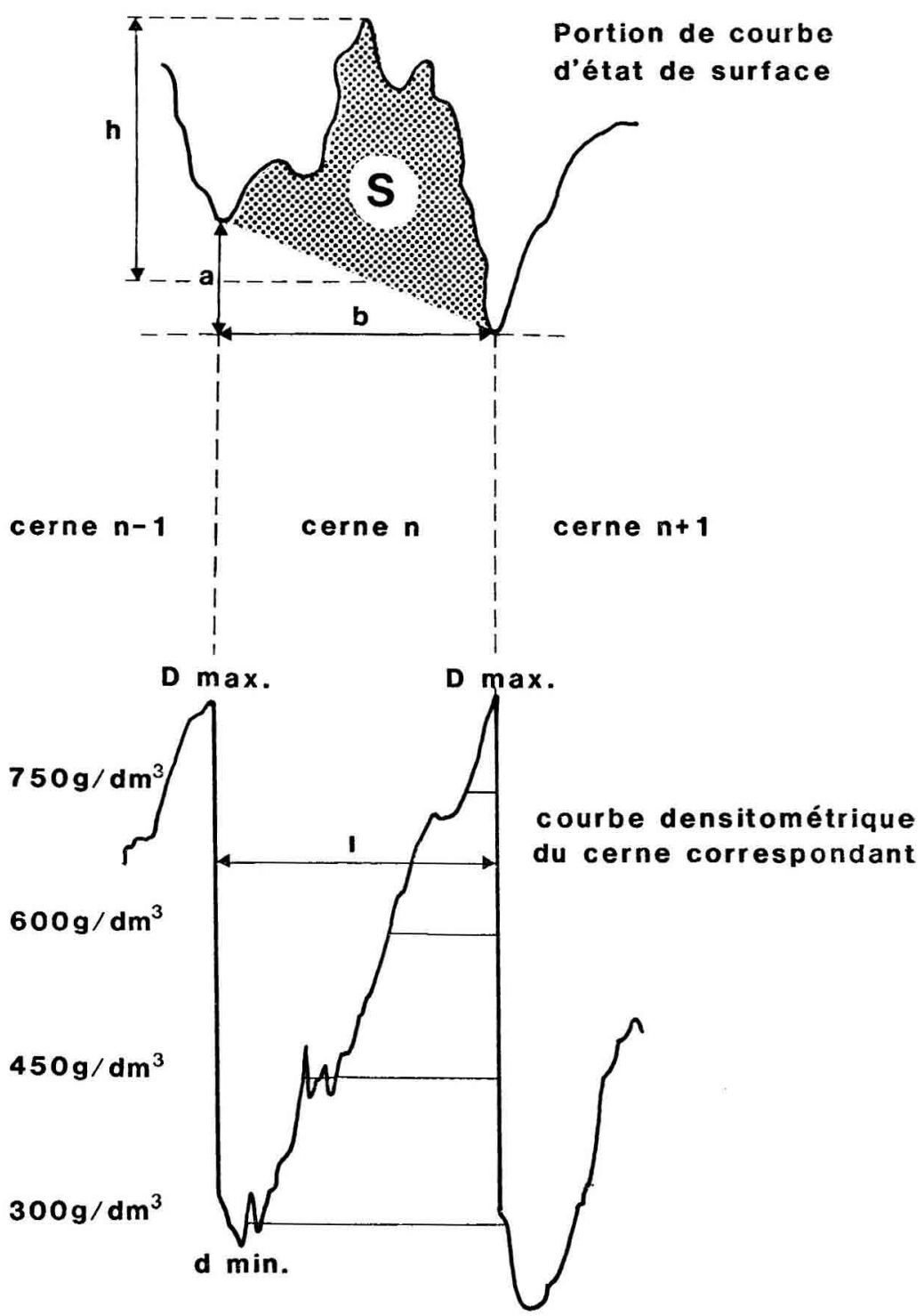

Fig. 3

Données relevées sur les portions de courbes d'état de surface et de courbes densitométriques des cernes correspondants.

Data measured on parts of smoothness and densitometric curves for corresponding rings. 
Il apparaît nettement que les «creux» importants de la courbe d'état de surface correspondent aux limites de cernes, donc aux variations brutales de densité d'un cerne à l'autre. Nous avons donc découpé la courbe d'état de surface en une série de portions de courbes dont les limites correspondent aux limites de cernes.

La figure 3 et le tableau 1 indiquent les critères qui ont été relevés sur les portions de courbes d'état de surface, ainsi que less composantes densitométriques notées cerne par cerne. Ces dernières ont été obtenues par traitement à lordinateur (KEI.I.r. \& Xeuxkt, 1973). Le tableau 2 décrit l'échantiilonnage retenu.

\section{TABI.EAU 1}

Données relevées à partir des portions de courbes d'état de surface et de courbes densitmétriques des cernes correspondants.

Data measured on parts of smoothness and densitometric curves for corresponding rings.

\begin{tabular}{|c|c|c|}
\hline Caractères & Unité & Symbole \\
\hline$-\mathrm{h}$ & !ı & $\mathrm{h}$ \\
\hline - $a / b \quad$ critères relevés sur les courbes d'état de surface & - & $a / b$ \\
\hline$-\mathrm{S} / \mathrm{b}$ & ! & $\mathrm{s} / \mathrm{b}$ \\
\hline -Densité minimum de cerne & $\mathrm{g} / \mathrm{dm} ;$ & \\
\hline - Densité maximum de cerne & $\mathrm{g} / \mathrm{dm}:$ & $D_{\max }$ \\
\hline - Densité moyenne de cerne & $\mathrm{g} / \mathrm{dm}: 3$ & $\mathrm{~d}_{\mathrm{ln}, y}$ \\
\hline — Largeur de cerne $\ldots \ldots \ldots \ldots \ldots \ldots \ldots \ldots \ldots \ldots \ldots$ & $\mathrm{mm}$ & largeur \\
\hline $\begin{array}{l}\text { - Hétérogénéité } 1: \text { densité maximum du cerne } n-\text { densité } \\
\text { minimum du cerne } n \ldots \ldots \ldots \ldots \ldots \ldots \ldots \ldots \ldots \ldots \ldots \ldots\end{array}$ & $\mathrm{g} / \mathrm{dm}^{3}$ & hétéro I \\
\hline $\begin{array}{l}\text { - Hétérogénéité } 2: \text { densité maximum du cerne }(n-1)- \\
\text { densité minimum du cerne } n \ldots \ldots \ldots \ldots \ldots \ldots \ldots \ldots\end{array}$ & $\mathrm{g} / \mathrm{dm}^{3}$ & hétéro 2 \\
\hline — Hétérogénéité 3: densité moyenne - densité minimum . . & $\mathrm{g} / \mathrm{dm}^{3}$ & hétéro 3 \\
\hline — Pourcentage de bois de densité supérieure à $300 \mathrm{~g} / \mathrm{dm}^{3}$.. & $\%$ & $\mathbf{d}_{3,11 !}$ \\
\hline _- Pourcentage de bois de densité supérieure à $450 \mathrm{~g} / \mathrm{dm}:$.. & $\%$ & $d_{4.0}$ \\
\hline - Pourcentage de bois de densité supérieure à $600 \mathrm{~g} / \mathrm{dm}^{3} \ldots$ & To & $d_{(i 00)}$ \\
\hline - Pourcentage de bois de densité supéricure à $750 \mathrm{~g} / \mathrm{dm}^{\prime \prime}$. & $\%$ & $d_{7 \pi n}$ \\
\hline données inversement proportionnelles à & $\%$ & $\mathrm{~d}_{3(1)-150}$ \\
\hline la pente de la courbe de densité entre les & $\%$ & $d_{15,(1-600}$ \\
\hline$-\mathrm{d}_{300}-\mathrm{d}_{600} \mid$ paliers considérés & $\%$ & $d_{3011-600}$ \\
\hline
\end{tabular}

- Pourcentage de bois de densité supérieure à $300 \mathrm{~g} / \mathrm{dm}^{*}$ - wood percentage with densily above $300 \mathrm{~g} / \mathrm{dr} \mathrm{m}^{\mathrm{s}}$.

- Paramètres inversement proportionnels à la pente de la courbe de densité entre les paliers considérés - Data inversely proporional to density curve slope between the considerated density levels. 
TABl_eaU 2

Répartition des 326 cernes étudiés suivant arbre et position dans la planche.

Sampling of 326 studied rings according to tree and position in the board.

\begin{tabular}{r|c|c|c|c|}
\hline \multirow{2}{*}{ Arbres } & \multicolumn{2}{|c|}{ Côté « départ » (indice d) } & Côté «arrivéc » (indice a) \\
\hline & Trajet 1 & Trajet 2 & Trajet 1 & Trajet 2 \\
\hline 2 & 10 & 10 & 12 & 12 \\
7 & 13 & 13 & 9 & 10 \\
8 & 13 & 12 & 13 & 12 \\
13 & 11 & 12 & 13 & 13 \\
21 & 9 & 8 & 10 & 8 \\
23 & 12 & 12 & 11 & 11 \\
28 & 11 & 11 & 9 & 9 \\
29 & - & - & & 9 \\
\hline
\end{tabular}

Le premier cerne pris en considération est toujours le second à partir de la moelle. Par excmple, l'arbre 13 mesuré còté départ sur le trajet 2 (indicé $13 \mathrm{~d} 2$ ) est étudié sur 12 cernes représentant les cernes d'âges 2 à 13 depuis la moelle.

The first considerated ring alu'ays is the secumd from the pith. For example, tree No. 13 measured on starting side for passage No. 2 (indexed 13 d 2) is studied for 12 rings corresponding to ages from 2 to 13 (from the pith).

\section{Remarques}

- La méthode adoptéc pour relever les critères de rugosité ne tient pas compte des ondulations de grande amplitude couvrant plusieurs cernes que l'on peut observer sur les courbes complètes, les moyens mathématiques nous faisant défaut. Il faut ajouter que ces ondulations peuvent être liées à la nature du bois, mais aussi à l'usure difŕférente des couteaux de la raboteuse le long de l'axe de l'arbre porte-outils.

- Nous avons également procédé à des radiographies tangentielles des parallélépipèdes de bois, mais nous avons constaté que la liaison état de surface-courbe densitométrique semblait meilleure avec les radiographies axiales ; c'est pourquoi nous avons poursuivi l'étude avec ces dernières.

Nous insisterons sur le fait que les courbes d'état de surface obtenues, comme toute courbe décrivant un étal de surface notamment en bois, sont dépendantes du protocole expérimental adopté (en particulier de la géométrie et de la pression du palpeur, laquelle interagit avec les variations de dureté du bois rencontrées). Dans le même esprit, nous indiquerons au lecteur que nous ne prétendons naturellement pas décrire intégralement les courbes d'état de surface à l'aide des 3 critères relevés.

\section{Liaisons recherchées. Calculs}

Nous avons déjà indiqué notre propos en introduction, mais de manière forcément imprécise du fait que la méthodologie n'était pas encore décrite.

\subsection{Liaisons étudiées}

Nous recherchons les liaisons suivantes: 


\subsection{Relation entre les composantes densitométriques d'un cerne particulier et les critères relevés sur la portion de courbe d'état de surface correspondant au cerne}

Pour ce faire, nous observons les corrélations intraindividu (l'individu étant défini par la population de cernes correspondant à un demi-trajet) ; celles-ci nous indiqueront dans quel sens évolue l'état de surface à l'intérieur d'un individu lorsqu'on modifie les composantes densitométriques en changeant d'accroissement annuel.

\subsection{Relation entre les composantes densitométriques moyennes d'un individu}

(l'individu étant défini comme ci-dessus) et ses critères d'état de surface moyens

Ellc va indiquer comment éventuellement évolue l'état de surface moyen si on modific les composantes densitométriques par sélection d'individus. C'est cette corrélation qui est la plus intéressante pour le sélectionneur.

Nous rendrons compte de ces deux types de liaison en ayant, au préalable, présenté la variabilité des caractères à l'intéricur des individus en fonction de l'âge, et la variabilité entre individus.

Il aurait été également intéressantu d'étudier les relations entre la variabilité des composantes densitométriques intraindividu (variabilité définie par exemple par leur écart-type) et, d'une part, des critères relevés sur les courbes d'état de surface moyens de l'individu, d'autre part, la variabilité de ces derniers. En effet. on pourrait considérer que des critères de rugosité trop variables à l'intérieur d'un individu sont néfastes au même titre que peuvent l'être des critères moyens de telle ou telle valeur. Malheurcusement notre échantillonnage était trop faible pour étudier raisonnablement ces liaisons.

\subsection{Mode de calcul}

Les points 2.11 et 2.12 ont été traités par un programme d'analyse de variancecovariance à un facteur qui, classiquement, permet de comparer des populations pour un certain nombre de caracteres, et de calculer les corrélations au niveau inter et intrapopulation.

l.e tableau 3 présente lanalyse de variance-covariance réalisée.

\section{TABLLAN 3}

Analyse de variance-covariance réalisée. Cas de l'échantillon comportant les cernes juvéniles. Variance and colariance analysis. Sampling with juvenile rings.

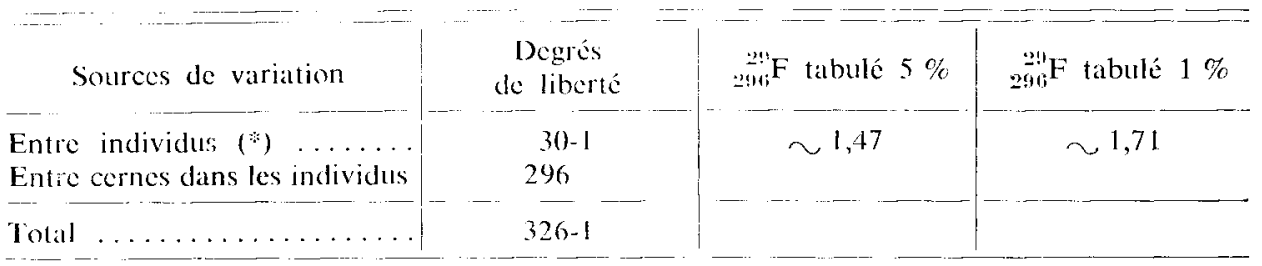

(*) Chaque demi-trajet est considéré comme un individu; il y a done cn tout 8 arbres $\times 2$ trajets $\times 2$ côtés (départ et arrivée) soir 32 «individus» -2 individus $=30 ; 1$ arbre 29 n'cst en effet pas représenté pour les trajets 1 et 2 , coté départ.

( $)$ Each half-passage is considerated as an individual; then there are 8 tres $\times 2$ passages $\times$ 2 sides (starting and arrival) let 32 «ndividuals» -2 individuals $=30$, because the tree No. 29

is only represented with passages No. 1 and 2, starting side.

- Degrés de liberté - Degrees of freedom.

- Entre individus - Between individials.

- Entre cernes dans les individus - Betreen rings in individaals. 


\section{Résultats}

\subsection{Variabilité des caractères en fonction de l'âge}

On a retenu uniquement pour cette étude 12 individus présentant au moins 10 cernes (c'est-à-dire des âges allant de 2 à 11 ans depuis la moelle), à savoir les individus $2 \mathrm{a} 1.7 \mathrm{a} 2.8 \mathrm{a} 1.13 \mathrm{a} 2,23 \mathrm{a} 1,28 \mathrm{a} 2,2 \mathrm{~d} 2,7 \mathrm{~d} 1,8 \mathrm{~d} 2,13 \mathrm{~d} 1,23 \mathrm{~d} 2,28 \mathrm{~d} 1$; on notera que les côtés "arrivée " et "départ ", ainsi que les trajets 1 et 2 sont répartis de manière équilibrée.

Les figures 4 et 5 présentent l'évolution de quelques variables, la figure 4 pour les composantes densitométriques. la figure 5 pour les critères d'état de surface. Des comparaisons de moyennes par séries appariées (au seuil de 5 p. 100) ont été effectuées pour tester le caractère significatif des variations dues à l'âge.

Si l'on excepte le cas de la largeur de cerne qui chute brutalement vers 7 ans, on remarque que les trois premiers cernes (c'est-à-dire ceux d'âges 2 à 4 depuis la moelle) se distinguent assez significativement des autres de la façon suivante : densité minimum de cerne élevée. densités moyenne et maximum de cerne relativement plus fortes (de manière toutefois moins nette que pour le premier caractère). Ces observations révélatrices d'un bois juvénile sont relativement conformes à celles notées sur Pseudotsuga menziesii. notamment par Polge (1964 et 1966, op. cit.) et Тнову (1975).

Le caractère singulier des cernes juvéniles apparaît également pour deux critères de rugosité (h et $\mathrm{S} / \mathrm{b}$ ), mais pas sur a/b malgré la très grande variabilité de ce dernier paramètre : $h$ et $\mathrm{S} / \mathrm{b}$ augmentent sensiblement en passant au bois « adulte».

Cette constatation nous a incités par la suite à effectuer certains calculs en excluant les cernes dont le caractère juvénile était plus prononcé (cernes d’âges 2 à 4 depuis la moelle), de manière à ne pas faire apparaître certaines liaisons ou à en masquer d'autres du simple fait de ce bois bien particulier.

\subsection{Variabilité individuelle des composantes densitométriques et des critères relevés sur les courbes d'état de surface}

Le tableau 4 rend compte du caractère plus ou moins significatif des différences individuelles, un individu étant, rappelons-le, la population de cernes correspondant à un des quatre demi-trajets d'état de surface effectués pour chaque planche.

Dans la $2^{\circ}$ colonne de ce tableau, on observe un effet individuel très important sur tous les caractères étudiés, tout particulièrement sur les densités maximales de cerne et, comme conséquence, sur les hétérogénéités 1 et $2\left({ }_{204}^{*: 1} \mathrm{~F}\right.$ de $12,74^{* *}$ à 16,70**), de même que sur la différence entre densité moyenne et densité minimum quii. est un autre critère d'hétérogénéité (hétérogénéité 3).

Viennent ensuite les $F$ «moyens" (de 4,12 à 7,40) pour la densité moyenne de cerne, les pourcentages de densité supérieure à certains niveaux, et les inverses des pentes entre ces derniers, ainsi que pour deux critères décrivant les états de surface : $h$ et $S / b$; enfin, les $F$ pour la largeur de cerne et $a / b$ avec des valeurs plus faibles, mais restant toutefois significatives au seuil de 1 p. 100. 


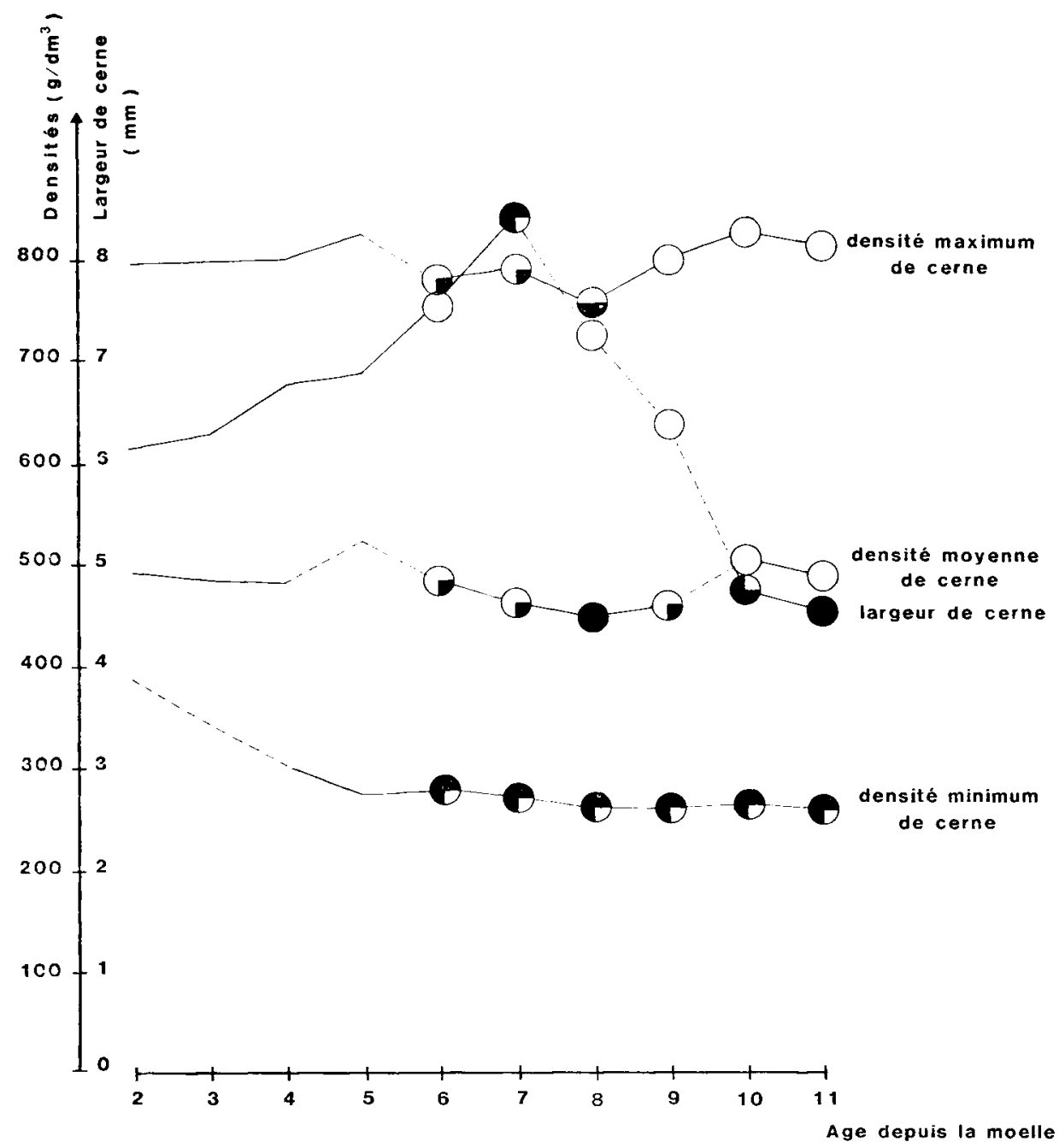

différence non significatives (seuil de $5 r_{i}$ ) entre cemes successifs - no significant difference (at $5 \%$ level) between sticcessive rings.

différences signilicatives (seuil de $5 \%$ ) entre cornes successifs - significant difference (at $5 \%$ level) between successite rings.

$3{ }^{2} \quad$ le cerne d'âge $n$ est différent des ccrnes d'âges 2 et 4 , mais non différent des cernes dages 3 at 5 - $n$ years old ring is differem from 2 and 4 years old rings, but no different from 3 and 5 years old rings.

FIG, 4

Variabilité de quelques composantes densitométriques avec l'âge.

Variability in terms of age for some densitometric components.

- Légende - Key.

- Largeur de cerne - Ring width.

- Densité maximum de cerne - Maximum ring density.

- Densité minimum de cerne - Minimum ring density.

- Densité moyenne de cerne - Mean ring density.

- Age depuis la moelle - Age from pith. 


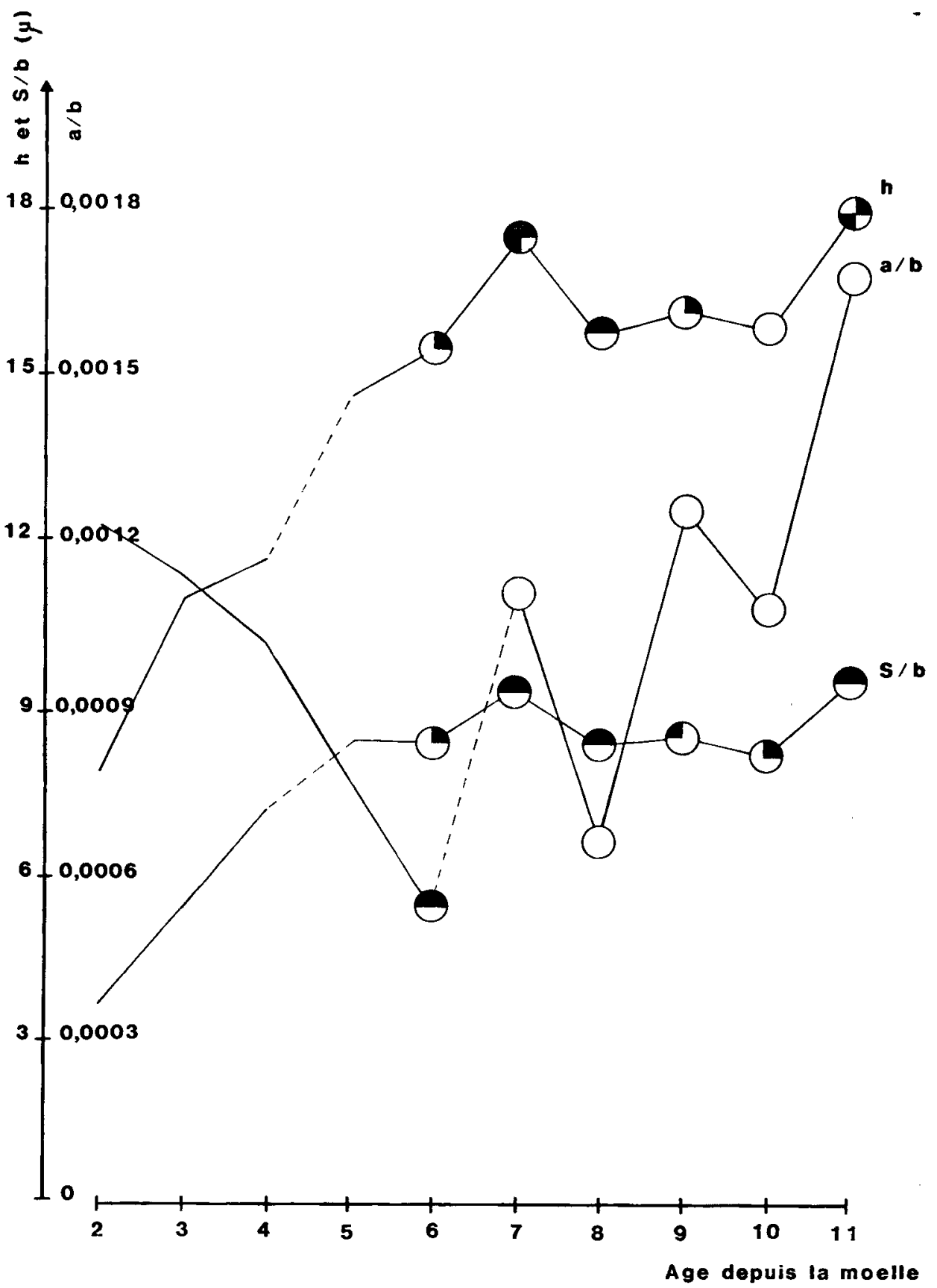

FIG. 5

Variabilité en fonction de l'âge des critères relevés sur les courbes d'état de surface (pour la légende: voir figure 4).

Variability in terms of age for criteria measured on smoothness curves. 


\section{TABII:AU 4}

Résultats des analyses de variance pour l'effet individuel sur les composantes densitométriques et les critères relevés sur les courbes d'état de surface.

Variance analysis results for individual effect on densitometric componemts and criteria measured on smoothness curves.

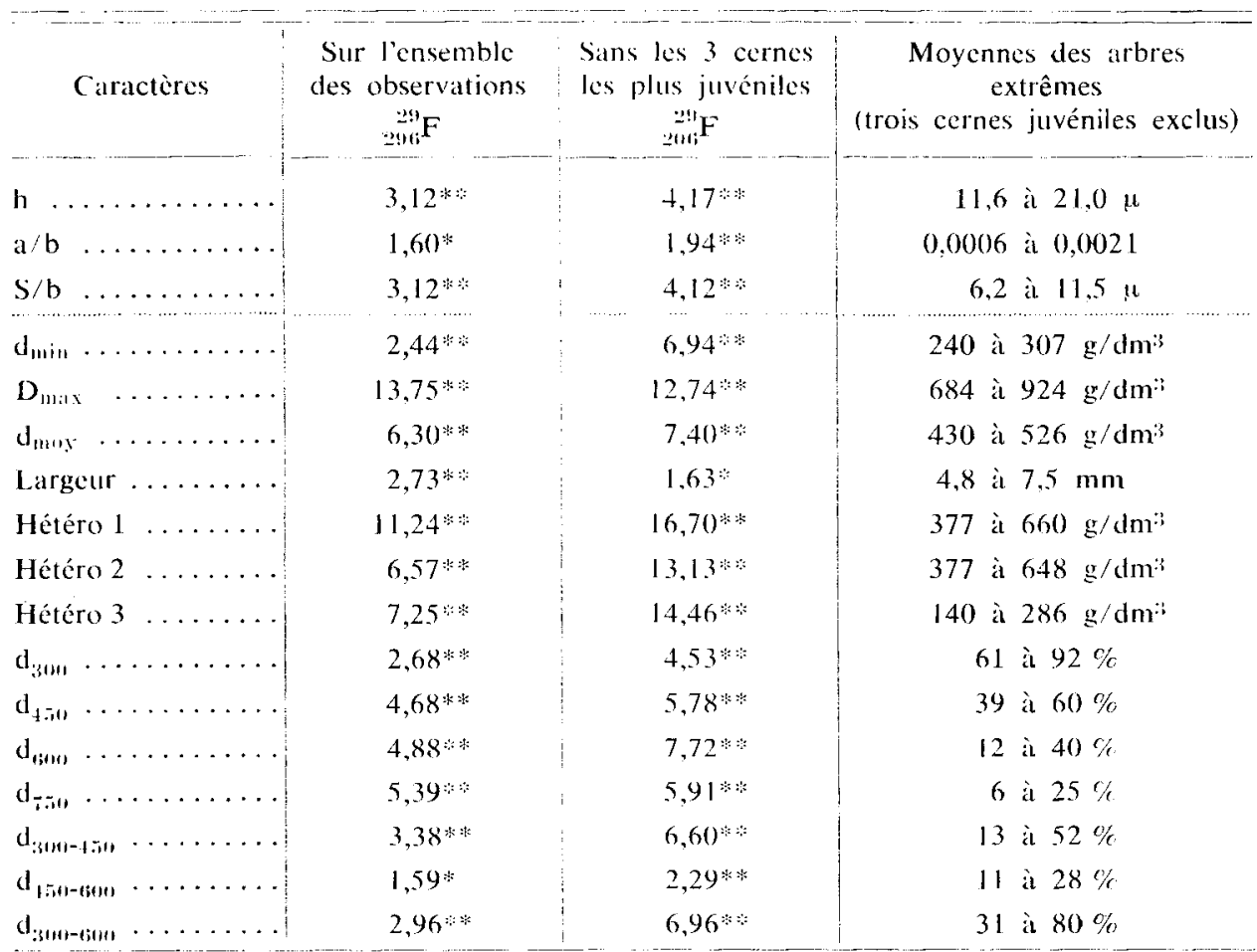

- Sur lensemble des observations - Sampling with all the rings.

- Sans les 3 cernes les plus juvéniles - Sampling withomt the 3 most jurenile rings.

- Moyennes des arbres extrêmes (trois cernes juvénilcs exclus) - Extreme trees means (nithout the 3 jurenile rings).

Nous constatons d'abord que le lait d'éliminer les trois cernes les plus juvéniles de notre échantillon (donc de diminuer la variabilité intraindividu) a pour effet d'augmenter les écarts entre individus pour tous les caractères mesurés (à l'exception de la largeur de cerne). De ce fail, nous poursuivrons une partie de nos interprétations, d'une part avec l'ensemble des observations, d'autre part avec un sous-ensemble issu de ce dernier et privé des trois cernes les plus proches de la moelle. De cette manière, il sera possible d'apprécier si les corrćlations éventuelles entre critères relevés sur les courbes d'état de surface et composantes densitométriques sont dues, ou non, à la présence de bois juvénile. 
Une question se pose immédiatement, qui conditionne l'intérêt d'une sélection individuclle (c'est-à-dire une sélection d'arbres) pour les caractères mesurés : quelle est l'importance de la variabilité intraarbre? En d'autres termes. les 4 trajets correspondant à chacun des 7 arbres éludiés (et 2 trajets pour l'arbre n" 29) sont-ils ou non très différents?

Les figures 6 à 11 répondent à cette question pour quelques-uns des critères étudiés (échantillons excluant les 3 cernes les plus proches de la moelle). Ces figures nous indiquent l'importance des différences, d'une part entre les trajets 1 et 2 . grâce à l'observation de la dispersion des points autour de la droite $y=x$, et d'autre part entre les côtés "arrivée » et "départ », c'est-à-dire les côtés opposés par rapport à la moelle.

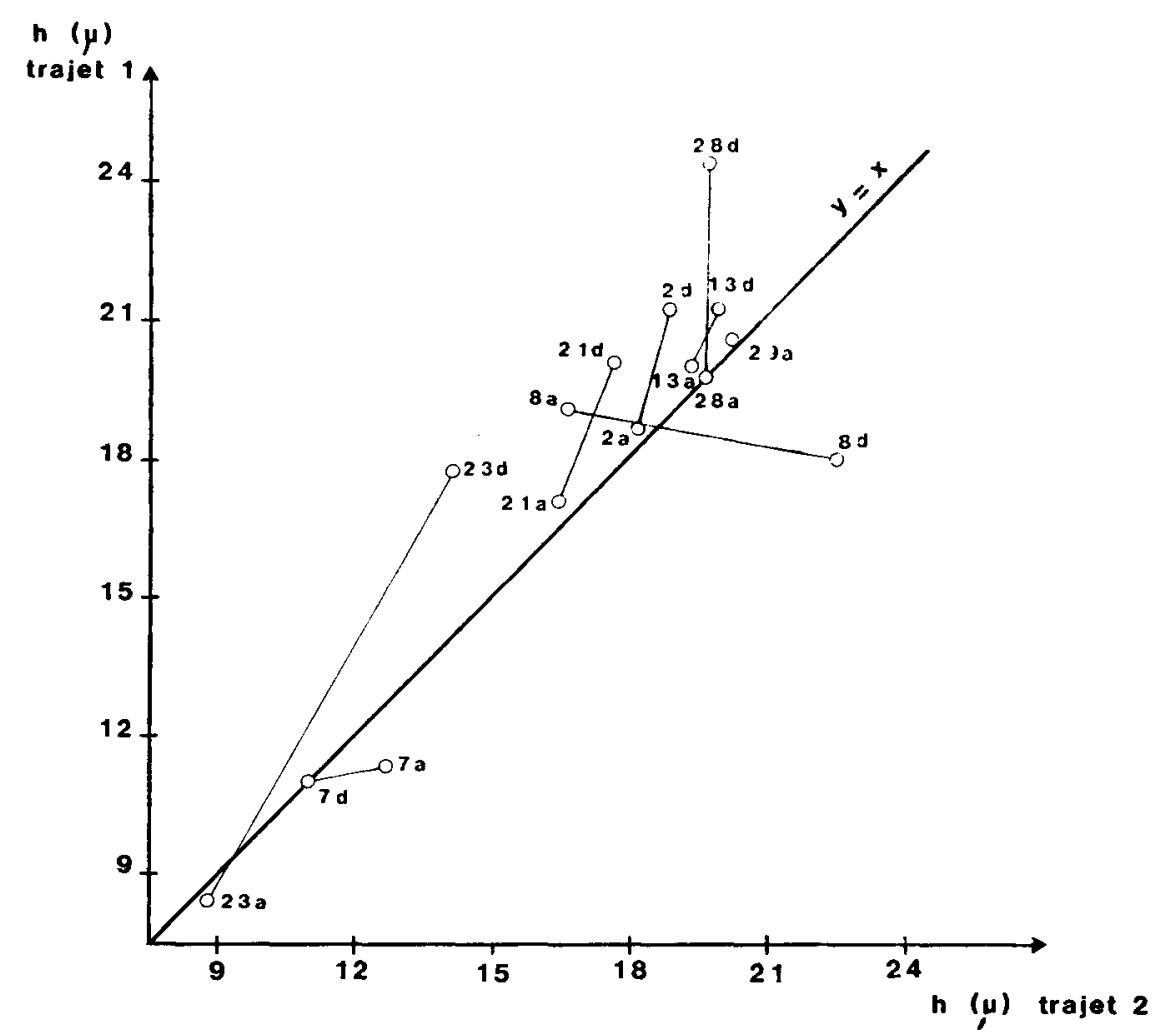

FIG. 6

Variabilité intraarbre des critères relevés sur les courbes d'état de surface : cas de $h$. Intratree variability for h criterion measured on smoothness curves.

- Echantillonnage sans bois juvénile - Sumpling without juvenile wood. 


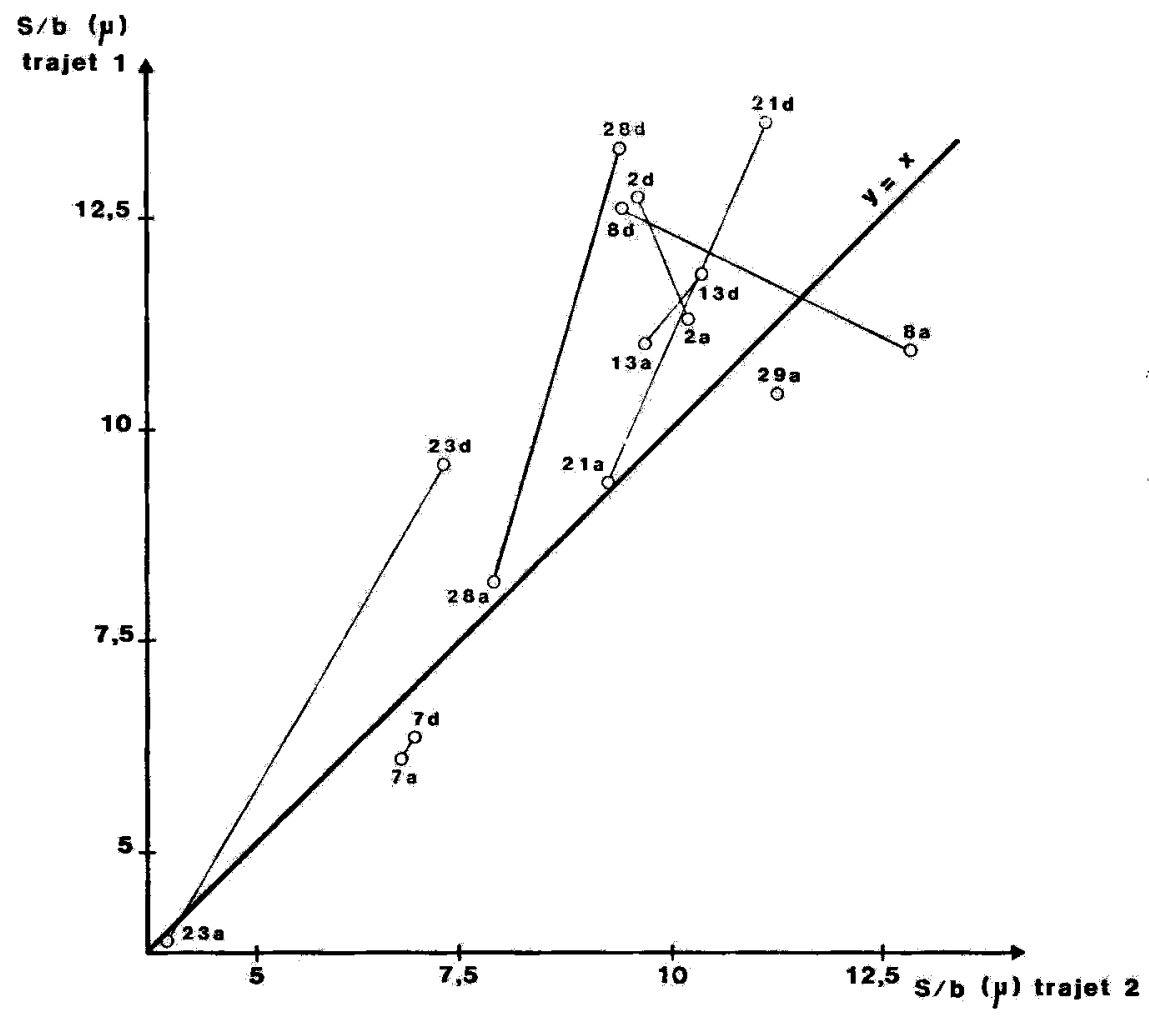

Fig. 7

Variabilité intraarbre des critères relevés sur les courbes d'état de surface ; cas de $S / b$. Intratree variability for $S / b$ criterion measured on smoothness curves.

- Echantillonnage sans bois juvénile - Sampling without juvenile wood. 


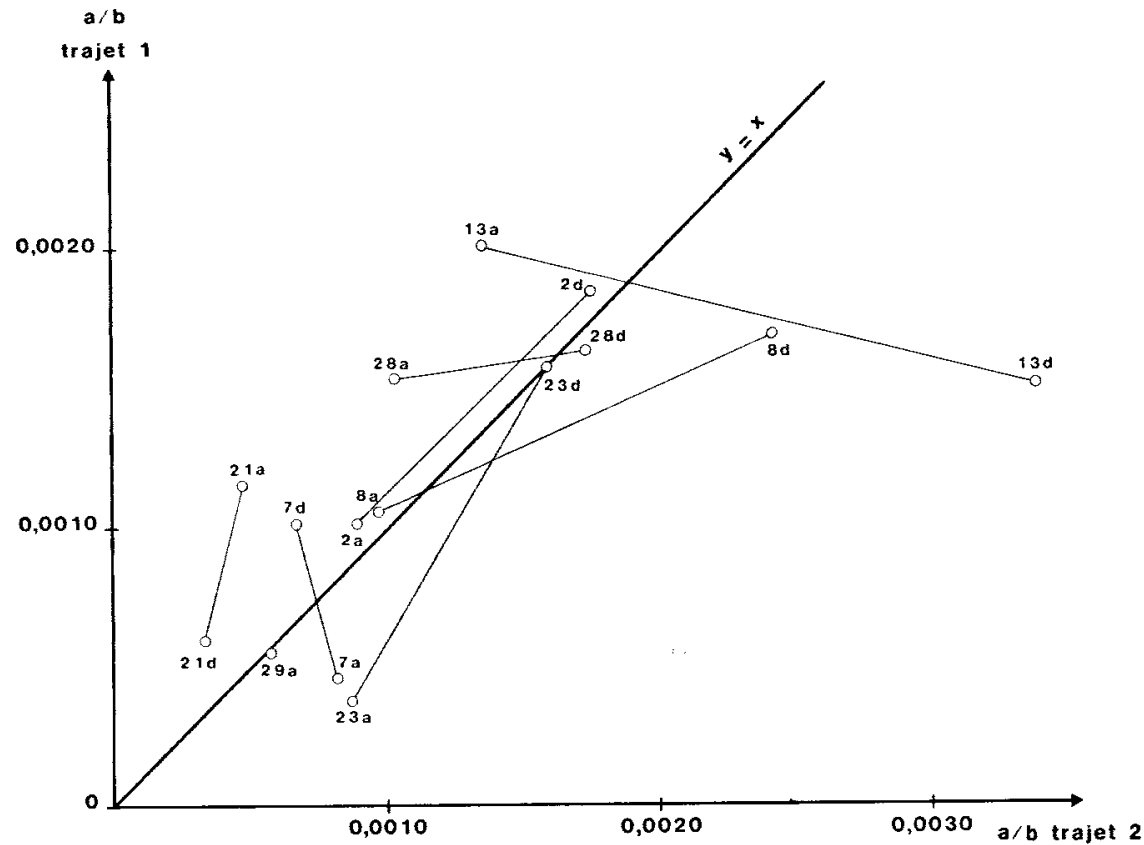

Fig. 8

Variabilité intraurbre des critères relevés sur les courbes d'éfat de surface : cas de a/b. Intratree variability for a/b criterion measured on smothness curves.

- Echantillonnage sans bois juvénile - Sampling without juveniie woret. 


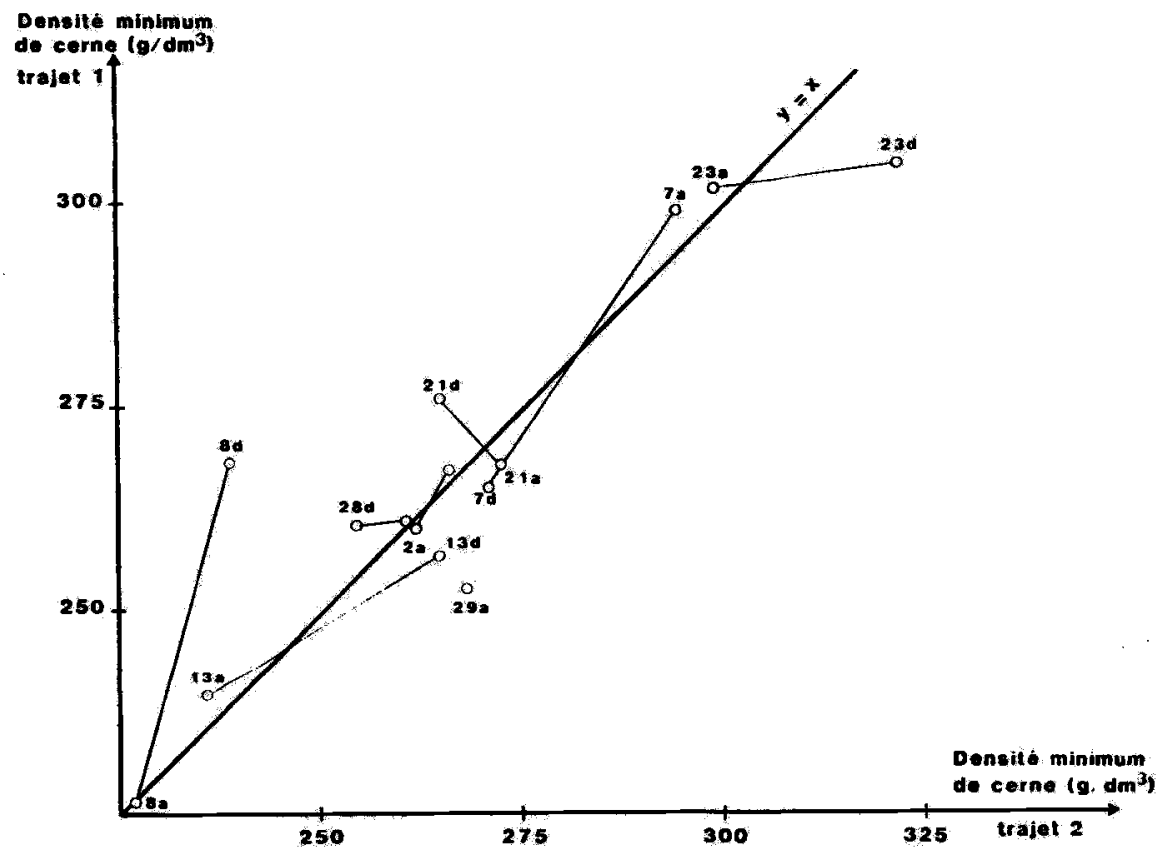

Fig. 9

Variabilité intraarbre des composantes densitométriques : cas de la densité minimum de cerne.

Intratree variability for minimum ring density.

- Echantillonnage sans bois juvénile - Sampling without juvenile wood. 


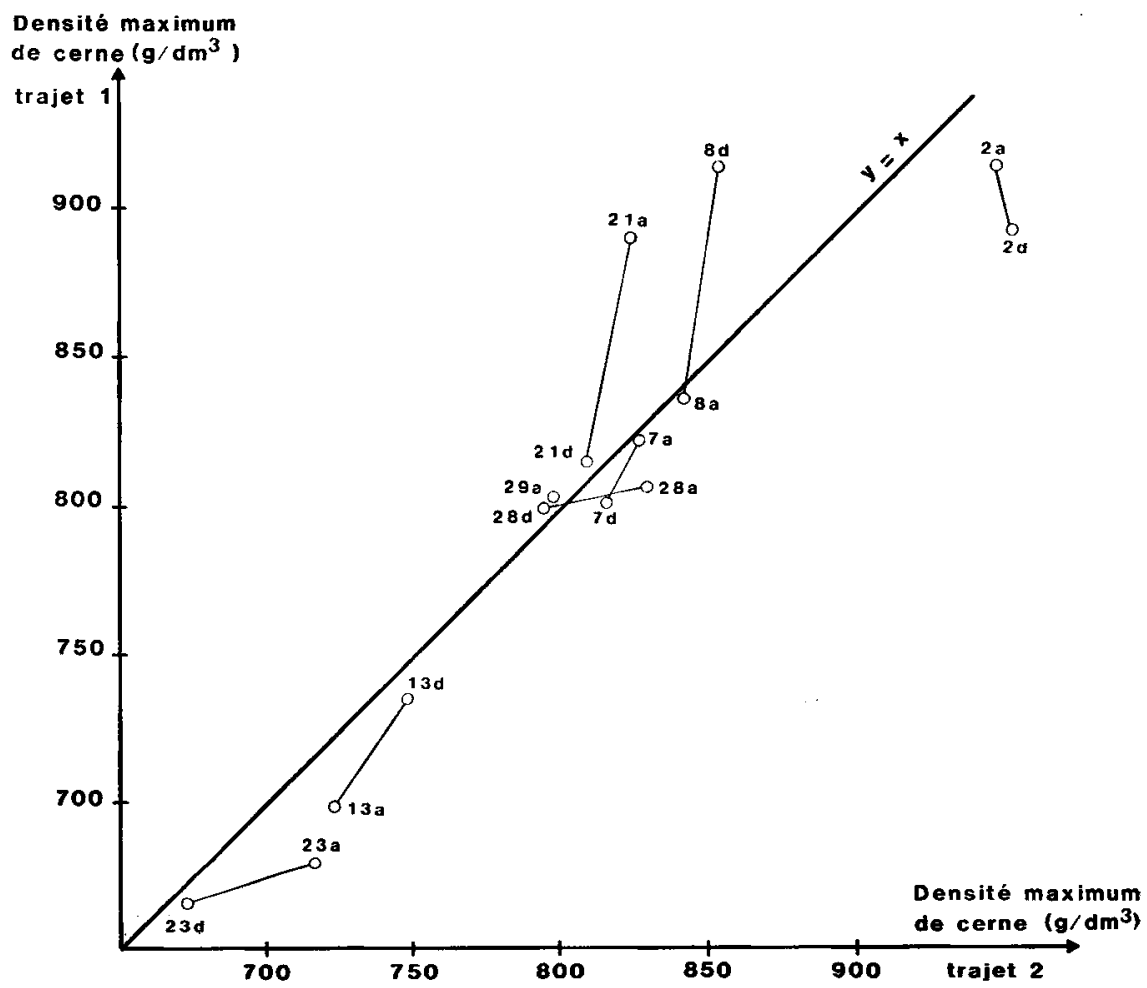

FIG. 10

Variabilité intraarbre des composantes densitométriques : cas de la densité maximum de cerne.

Intratree variability for maximum ring density.

- Echantillonnage sans bois juvénile - Sampling without juvenile wood. 


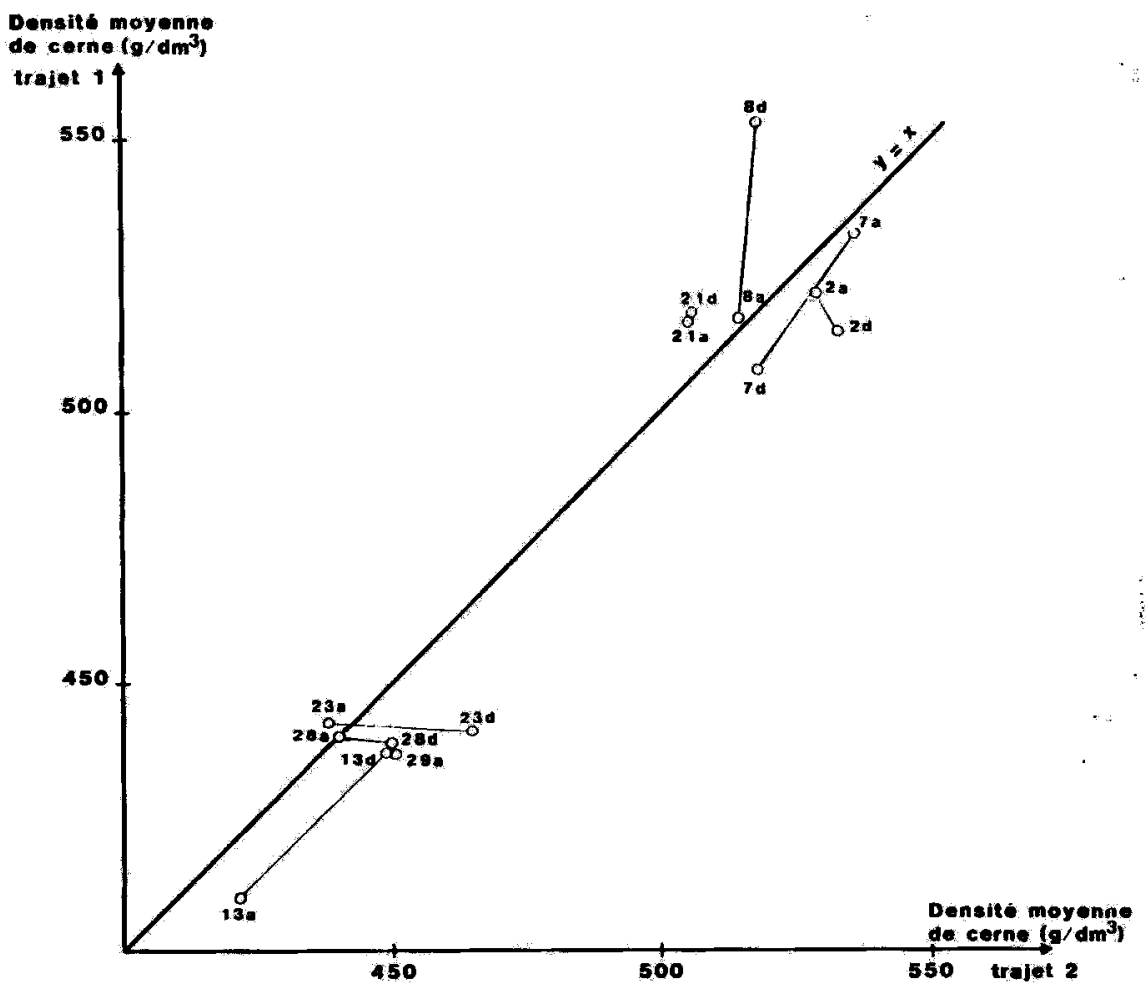

Fig. 11

Variabilité intraarbre des composantes densitomêtriques : cas de la densité moyenne de cerne.

Intratree variability for mean ring density.

- Echantillonnage sans bois juvénile - Sampling without jwvenile wood. 
A l'exception de a/b, il apparaît que les trajets 1 et 2 sont relativement identiques pour tous les caractères ( $h, S / b$ et composantes densitométriques), et ce malgré le fait que toutes les populations ne présentent pas strictement le même nombre de cernes, donc pas les mêmes années calendaires. Ceci veut dire que la variabilité est faible le long d'une génératrice de l'arbre et que les néthodologies de mesure employées dans cette expérience sont fiables.

Il convient aussi de signaler que les critères d'états de surface h et $S / b$ (fig. 6 et 7) sembleraient être moins bons pour les trajets 1 que pour les trajets 2 , puisque la plupart des points sont au-dessus de la droite $y=x$. Ceci pourrait signifier que, du point de vue de ces 2 paramètres, les états de surface sont moins bons à la partie inférieure des arbres (le trajet 1 étant toujours situé plus bas que le trajet 2 ; voir fig. 1) ou plus probablement à une question de comportement de l'outil, lequel travaillait toujours du trajet 2 vers le trajet 1 .

Si maintenant on passe d’un côté à l'autre de la moelle, en suivant le même diamètre de l'arbre, c'est-à-dire si l'on compare les côtés a (arrivée) et d (départ), on constate des cas de figure assez différents suivant les caractères. Pour la densité maximum et moyenne de cerne (fig. 10 et 11 ), on note une stabilité relative. Tel n'est pas le cas pour la densité minimum de cerne (fig. 9) et les 2 critères $h$ et $S / h$ (fig. 6 et 7), du moins en ce qui concerne certains arbres (arbre n" 23 pour h et S/b, arbre $\mathrm{n}^{\circ} 8$ pour la densité minimum), alors que d'autres sont particulièrement stables (arbres $\mathrm{n}^{\circ:} 2$ et 13 pour $\mathrm{h}$ et $\mathrm{S} / \mathrm{b}$, arbres $\mathrm{n}^{(: 4} 2,21$ et 28 pour la densité minimum). Ceci illustre le fait déjà bien connu que les arbres, s'ils sont différents du point de vue de lcurs propriétés moyennes, le sont aussi pour la variabilité de ces mêmes propriétés; il n'est d'ailleurs pas à exclure que des critères d'homogénéité intraarbre soient aussi, sinon plus, importants pour l'aptitude technologique que la moyenne individuelle de ces critères.

Notons enfin, tant pour $h$ que pour $S / b$, que les côtés «départ » ne présentent pas de valeurs systématiquement supérieures ou inférieures aux côtés «arrivée»; c'est un point positif pour le rugosimètre utilisé qui semble donc ne pas donner un biais systématique suivant le sens de mesure (de l'écorce vers le cœur pour le côté "départ» ou du coeur vers l'écorce pour le côté «arrivée »).

\subsection{Relations intraindividu entre composantes densitométriques et critères relevés sur les courbes d'état de surface}

Le tableau 5 présente ces corrélations en distinguant l'échantillonnage complet et celui privé des 3 cernes à caractère juvénile. Les corrélations sont mutipliées par 1000 . Il apparaît des liaisons nettement significatives entre les critères $h$ et $\mathrm{S} / \mathrm{b}$ d'une part, la densité minimum d'autie part (plus la densité minimum est forte, meilleur est l'état de surface). En revanche, ni la densité moyenne de cerne, ni la densité maximum de cerne ne semblent influencer les critères de rugosité ainsi définis. Les coefficients significatifs et positifs avec les 3 critères d'hétérogénéité hétéro 1 , hétéro 2 et hétéro 5 , ainsi qu'avec le pourcentage de bois de densité supérieure à $300 \mathrm{~g} / \mathrm{dm}^{3}\left(\mathrm{~d}_{30 m}\right)$ résultent vraisemblablement de l'effet de la densité minimum qui sert à les calculer. Nous observons également une relation linéaire élevée de $\mathrm{h}$ et de

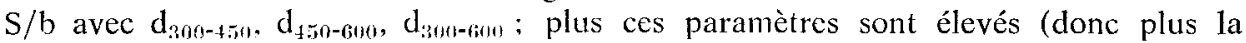
pente de la courbe densitométrique intracerne est faible), plus $h$ et $\mathrm{S} / \mathrm{b}$ semblent donc s'améliorer. 
TABLEAL 5

Liaisons intraindividus entre critères releves sur les courbes d'état de surface et composantes densitométriques (corrólations multipliées par 1000$)$.

Intraindividal relationships between criteria measured on smoothness curves and densitometric components (the correlations are maltiplied by I 000).

\begin{tabular}{|c|c|c|c|c|c|c|}
\hline \multirow{2}{*}{$\begin{array}{r}\begin{array}{r}\text { Critères relevés } \\
\text { sur les courbes } \\
\text { d'état de } \\
\text { surface }\end{array} \\
\text { Composantes } \\
\text { densitométriques }\end{array}$} & \multicolumn{2}{|c|}{ h } & \multicolumn{2}{|c|}{$a / b$} & \multicolumn{2}{|c|}{$\mathbf{S} / \mathbf{b}$} \\
\hline & 1 & 2 & 1 & 2 & 1 & 2 \\
\hline $\mathrm{d}_{\mathrm{min}}$ & $-556^{2 * 2 k}$ & $-318 * *$ & $-70 \mathrm{NS}$ & $-28 \mathrm{NS}$ & $-519^{* *}$ & $-308 * *$ \\
\hline $\mathbf{D}_{\text {max }} \ldots$ & $102 \mathrm{NS}$ & $44 \mathrm{NS}$ & $120^{* *}$ & $195 *$ & $77 \mathrm{NS}$ & $-21 \mathrm{NS}$ \\
\hline$d_{m}, \ldots$. & $-119 *$ & $-102 \mathrm{NS}$ & $101 \mathrm{NS}$ & $154^{*}$ & $-51 \mathrm{NS}$ & $-68 \mathrm{NS}$ \\
\hline l.argeur & $-70 \mathrm{NS}$ & $-100 \mathrm{NS}$ & $-320 *$ & $-413^{* * *}$ & - $75 \mathrm{NS}$ & $-88 \mathrm{NS}$ \\
\hline Hétéro I & $436 * *$ & $174^{*}$ & $1.37 \%$ & $209^{* * *}$ & $393 *$ & $103 \mathrm{NS}$ \\
\hline Hétéro 2 & $457^{* *}$ & $216^{* *}$ & $95 \mathrm{NS}$ & $161 *$ & $448 * *$ & $178^{* * *}$ \\
\hline Hétéro 3 & $431^{* *: 3}$ & $89 \mathrm{NS}$ & $159 *$ & $210^{* * *}$ & $456 * *$ & $125 \mathrm{NS}$ \\
\hline $\mathrm{d}_{: 3, \ldots,}$ & $-564 * *$ & $-329 * *$ & $-157 * *$ & $-150^{*}$ & $-497 * *$ & $-264 * *$ \\
\hline$d_{4 \ldots+1}$ & - $18 \mathrm{NS}$ & - $89 \mathrm{NS}$ & $57 \mathrm{NS}$ & $35 \mathrm{NS}$ & $92 \mathrm{NS}$ & $67 \mathrm{NS}$ \\
\hline$d_{f i o n}$ & $273 * *$ & $91 \mathrm{NS}$ & $151 *:$ & $223 * *$ & $295 * *$ & $76 \mathrm{NS}$ \\
\hline $\mathrm{d}_{i 51} \ldots$ & $199^{* * *}$ & $27 \mathrm{NS}$ & $249 *:$ & $293 * *$ & $167 *$ & - $46 \mathrm{NS}$ \\
\hline $\mathrm{d}_{30(1)-1, \ldots o}$ & $-499 * *$ & $---2.72 *$ & $-181 *:$ & $-186 * *$ & $-514 * *$ & $-330^{* *}$ \\
\hline $\mathrm{d}_{+\sin (1 ; 00)}$ & $-298 * *$ & $-175 \%$ & $-97 \mathrm{NS}$ & $-188^{* *}$ & $-211^{* *}$ & - $10 \mathrm{NS}$ \\
\hline $\mathrm{d}_{3011-10101} \ldots \ldots$ & $-562 * *$ & $-332^{4: 5}$ & $-198 \mathrm{NS}$ & $-273^{* * 1 ;}$ & $-527^{* * *}$ & $-269 *$ \\
\hline \multicolumn{7}{|c|}{$\begin{array}{l}\text { I : Echantillonnage complet - Whole sampling. } \\
2 \text { : Echantillonnage excluant les } 3 \text { cernes les plus juvéniles - Sampling wirhout the } 3 \text { most jurenile } \\
\text { rings. }\end{array}$} \\
\hline \multicolumn{7}{|c|}{$\begin{array}{l}\text { Avec l'échantillonnage complet ( } 326 \text { cernes), la corrélation intraindividu a } 296 \text { degrés de liberté. } \\
\text { Avec l'échantillonnage excluant les } 3 \text { cernes les plus juvéniles, la corrélation a } 206 \text { degrés de liberté. }\end{array}$} \\
\hline $\begin{array}{l}\text { With the whole sampling ( } 326 \\
\text { With sampling without the } 3\end{array}$ & $\begin{array}{l}\text { rings), the } \\
\text { most julent }\end{array}$ & $\begin{array}{l}\text { iniraindivic } \\
\text { mile rings, }\end{array}$ & $\begin{array}{l}\text { lual correlat } \\
\text { he correlatic }\end{array}$ & $\begin{array}{l}\text { tion has } 29 \\
\text { ion has } 206\end{array}$ & 6 degrees 6 & $\begin{array}{l}\text { of freedom. } \\
\text { of freedom. }\end{array}$ \\
\hline
\end{tabular}

L'élimination de l'échantillonnage des 3 cernes les plus juvéniles conduit pour $h$ et $S / b$ à une chute globale des cocfficients; ceci s'explique très bien par le fait que les cernes juvéniles ont le bois à densité minimum la plus forte et des critères $h$ et $\mathrm{S} / \mathrm{b}$ relativement moins élevés (fig. 4 et 5 ). Ces cernes contribuent donc assez fortement aux variabilités et aux corrélations intraindividuelles dont il est ici question. Nous soulignerons cependant que, malgré cette élimination, les coefficients restent statistiquement significatifs.

Le critère $a / b$ se comporte assez différemment des 2 autres critères décrivant l'état de surface; en premier lieu, presque tous les cocfficients augmentent en valeur absolue après élimination du bois juvénile. On ne retrouve pas la relation avec la densité minimum, mais un coefficient positif et (faiblement) significatif avec 
les densités moyenne et maximum de cerne (a/b augmenterait, donc l'état de surface se dégraderait quand ces deux composantes densitométriques augmenteraient).

Les relations avec les pourcentages de densité supérieure à $600 \mathrm{~g}$ et $750 \mathrm{~g} / \mathrm{dm}^{3}$, ainsi qu'avec les critères d'hétérogénéité résultent des observations ci-dessus. On note également une amélioration de l'état de surface (du point de vue de a/b) pour les cernes les plus larges.

\subsection{Relations interindividus entre composantes densitométriques et critères relevés sur les courbes d'état de surface}

Le tableau 6 rend compte de ces relations. Il apparaît cohérent avec le tableau 5 . On remarque en particulier une forte corrélation négative entre $h$ et $S / b$ d'une part, la densité minimum de cerne d'autre part ; la figure 12 illustre cette dernière pour la relation entre $d_{\text {min }}$ et $h$. On peut constater qu'en améliorant la densité minimum de cerne au niveau individuel de $65 \mathrm{~g} / \mathrm{dm}^{3}$, ce qui correspond à l'ordre de grandeur de la variabilité interarbre (voir tabl. 4), on peut faire décrôtre $h$ de 7 l , soit une amélioration de presque 40 p. 100 par rapport à une movenne générale de 18 fl.

\section{TABLEAU 6}

Liaisons interindividus entre critères relevés sur les courbes d'état de surface et composantes densitométriques (corrélations multipliées par 1000).

Interindividuals relationships between criteria measured on smoothness carres and densitometric components (the correlations are multiplied by 1000 ).

\begin{tabular}{|c|c|c|c|c|c|c|}
\hline \multirow[b]{2}{*}{$\begin{array}{l}\text { Composantes } \\
\text { densitométriques }\end{array}$} & \multicolumn{2}{|c|}{$\mathrm{h}$} & \multicolumn{2}{|c|}{$a / b$} & \multicolumn{2}{|c|}{$S / b$} \\
\hline & 1 & 2 & 1 & 2 & 1 & 2 \\
\hline$d_{\text {min }}$ & $-635^{* *}$ & $-557^{*}:$ & $-207 \mathrm{NS}$ & - $191 \mathrm{NS}$ & $-652^{* * *}$ & $-534 * *$ \\
\hline $\mathbf{D}_{\text {max }}$ & $230 \mathrm{NS}$ & $21.1 \mathrm{NS}$ & - $88 \mathrm{NS}$ & $-113 \mathrm{NS}$ & $320 \mathrm{NS}$ & $333 \mathrm{NS}$ \\
\hline $\mathrm{d}_{\mathrm{m} w \mathrm{y}}$. & $-247 \mathrm{NS}$ & $-208 \mathrm{NS}$ & $-256 \mathrm{NS}$ & $-224 \mathrm{NS}$ & $34 \mathrm{NS}$ & 109 NS \\
\hline Largeur & $-160 \mathrm{NS}$ & $-338 \mathrm{NS}$ & $-671^{* * *}$ & $-524 * *$ & $-106 \mathrm{NS}$ & $-288 \mathrm{NS}$ \\
\hline Hétéro 1 & $385 *$ & $332 \mathrm{NS}$ & $-12 \mathrm{NS}$ & $-49 \mathrm{NS}$ & $467 * *$ & $434 *$ \\
\hline Hétéro 2 & 347 NS & $288 \mathrm{NS}$ & $-17 \mathrm{NS}$ & - $84 \mathrm{NS}$ & $424 *$ & $398 *$ \\
\hline Hétéro 3 & $150 \mathrm{NS}$ & $77 \mathrm{NS}$ & $\ldots 96 \mathrm{NS}$ & $-108 \mathrm{NS}$ & $394 *$ & $347 \mathrm{NS}$ \\
\hline $\mathrm{d}_{30010} \ldots$ & 一 $650 * *$ & $-631^{* *}$ & $-303 \mathrm{NS}$ & $-259 \mathrm{NS}$ & $-512^{* * *}$ & $-452 *$ \\
\hline $\mathrm{d}_{4,50}$ & - $287 \mathrm{NS}$ & $-235 \mathrm{NS}$ & $-211 \mathrm{NS}$ & $-172 \mathrm{NS}$ & $37 \mathrm{NS}$ & $117 \mathrm{NS}$ \\
\hline $\mathrm{d}_{6 ; 00}$ & $-78 \mathrm{NS}$ & $-29 \mathrm{NS}$ & $-272 \mathrm{NS}$ & $-226 \mathrm{NS}$ & $153 \mathrm{NS}$ & $220 \mathrm{NS}$ \\
\hline $\mathrm{d}_{7.51} \ldots$ & $174 \mathrm{NS}$ & $175 \mathrm{NS}$ & - $\quad 4 \mathrm{NS}$ & - $25 \mathrm{NS}$ & $363^{*}$ & $383 *$ \\
\hline $\mathrm{d}_{3(3) 1-1501} \cdots \ldots \ldots$ & $-294 \mathrm{NS}$ & $-368 *$ & $-75 \mathrm{NS}$ & $-94 \mathrm{NS}$ & $-443 *$ & $-480^{*}$ \\
\hline$d_{450-609} \ldots \ldots \ldots \ldots$ & -352 NS & $-303 \mathrm{NS}$ & $129 \mathrm{NS}$ & $153 \mathrm{NS}$ & $-212 \mathrm{NS}$ & $-225 \mathrm{NS}$ \\
\hline$d_{3,4(1)-6,00} \ldots \ldots \ldots \ldots \ldots$ & $-393^{*}$ & $\mid--400^{*}$ & $-13 \mathrm{NS}$ & $-14 \mathrm{NS}$ & $-465^{* *}$ & $-456^{*}$ \\
\hline
\end{tabular}

La corrélation interindividus a 29 degrés de liberté.

The intertree correlation has 29 degrees of freedom.

1 : Echantillonnage complet - Whole sampling.

2 : Echantillonnage excluant les 3 cernes les plus juvéniles - Sampling without the 3 most juvenile rings. 


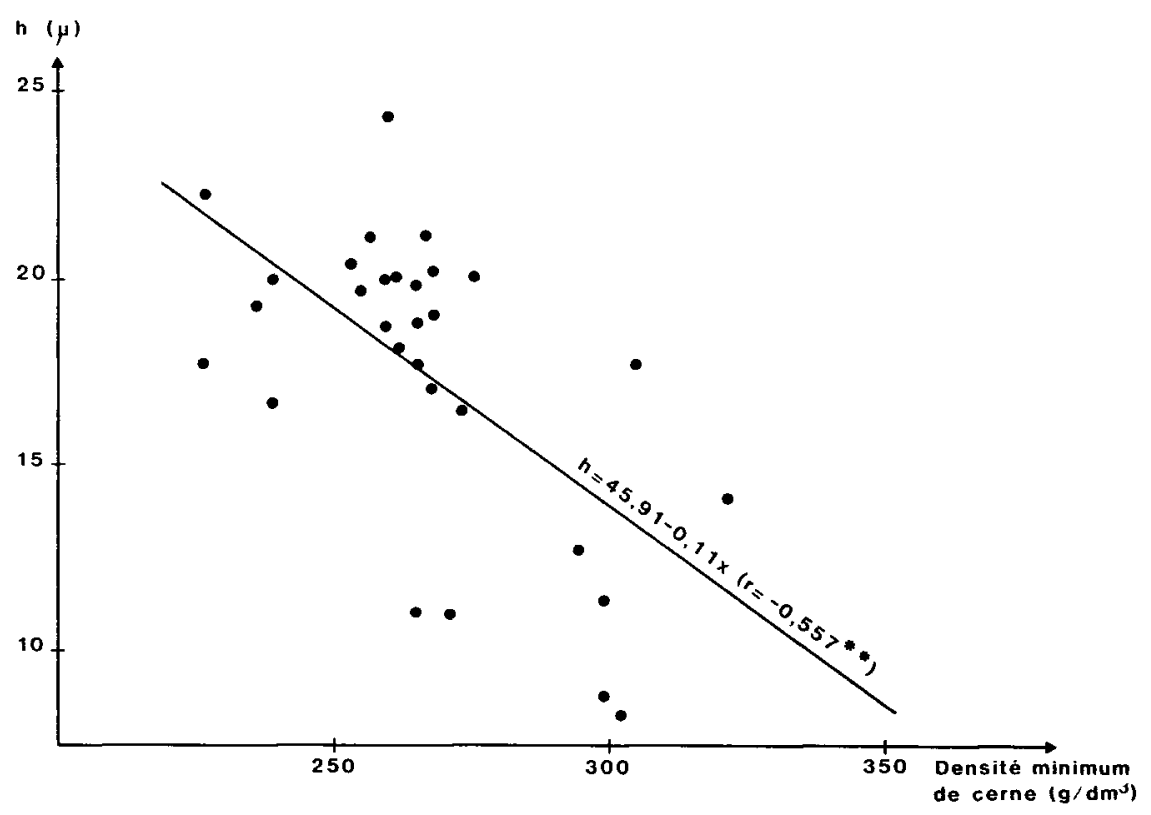

FIg. 12

Effet de la densité minimum de cerne sur le critère de rugosité h.

Minimum ring density effect on h smoothess criterion.

- Niveau individuel - Intertree level.

- Echantillonnage sans bois juvénile - Sampling without juvenile rood.

\section{Discussion et conclusions}

Le travail présenté ici apporte un certain nombre d'informations relativement inćdites qu'il serait important de confirmer avec un échantillonnage plus étendu et des moyens matériels qui nous faisaient défaut jusqu'à présent. Il serait en particulier nécessaire de réfléchir davantage aux critères d'état de surface que nous n'avons pu appréhender ici que de manière grossière, d'où, sans nul doute, un appauvrissement notable de l'information, de même qu'aux techniques de mesure et de saisie de ces importantes propriétés technologiques.

Cette remarque vaut aussi pour l'information recueillie sur les courbes densitométriques, encore que dans ce cas, les moyens matériels soient maintenant disponibles (Perrin \& Ferrand, 1983).

L'utilisation de techniques améliorées devrait permettre d'affiner plus encore les résultats intéressants dont nous disposons d'ores et déjà et qui nous conduisent à avancer les affirmations suivantes : 
- le bois juvénile présente des propriétés originales sur le plan de la rugosité, propriétés qui s'expliquent pour une large part du fait des profils densitométriques particuliers de ce bois ;

- il existe une variabilité individuelle significative pour la qualité des états de surface de planches rabotées à l'intérieur de l'espèce Douglas ;

- l'état de surface est lié, tant à l'intérieur des individus (en passant d'un cerne à l'autre) qu'entre individus, aux composantes densitométriques que l'on peut relever par radiographie d'échantillons non destructifs (carottes de sondage);

- parmi ces composantes densitométriques, la densité minimum de cerne est un critère déterminant; on peut estimer qu'un gain de $65 \mathrm{~g} / \mathrm{dm}^{3}$, réaliste du fait de la variabilité individuelle de ce caractère, pourrait conduire à une amélioration de près de 40 p. 100 du critère de rugosité étudié.

\section{Summary \\ Relationships between densilometric components and planed boards smoothness in Pseudotsuga menziesii}

Three criteria were determined on smoothness curves of planed boards from 8 young douglas fir (Pseudotsuga menziesii). The relationships between these criteria and densitometric components (measured by X-ray technique) distinguishing intratree variability (between rings) and individual variability (between trees) were studied.

The main results are the followings :

- juvenile wood smoothness parameters are particular with respect to mature wood. This fact seems to be related to densitometric components of juvenile wood:

- the individual variability is significant for planed boards smoothness;

- at intra- and intertree levels, the smoothness is related to densitometric components ;

- among these densitometric components, the minimum ring density is a determinant criterion; we estimated that an increase of $65 \mathrm{~kg} / \mathrm{m}^{3}$ for this characteristic can improve smoothness by an average of 40 percent.

\section{Zusammenfassung}

\section{Zusammenhänge zwischen Dichtekomponenten und der Oberflächenrauhigkeit gehobelter Brelter der Douglasie (Pseudotsuga menziesii)}

Drei Kriterien wurden bei den Oberflächenrauhigkeitskurven gehobelter Bretter an acht Stämmen junger Douglasien unterschieden. Anschliessend wurden die Zusammenhänge zwischen diesen Kriterien und den an Hand von Röntgenaufnahmen ermittelten Dichtkomponenten untersucht. Dabei wurde die Variabilität innerhalb der Bäume d.h. zwischen den Jahrringen und zwischen den Bäumen berücksichtigt.

Die wichtigsten Ergebnisse können wic folgt zusammengefasst werden :

- das Holz junger Douglasien zeigt spezielle Rauhigkeitskriterien dic durch die speziellen Dichtekomponenten dieses Holzes erklärt werden können; fikant ;

- die individuelle Variabilität der Oberflächenrauhigkeit gehobelter Bretter ist signi- 
- die Oberflächenrauhigkeit innerhalb und zwischen den Bäumen ist mit den Dichtekomponenten korreliert;

- unter den Dichtekomponenten ist vor allem das Dichteminimum der Jahrringe ein wesentliches Merkmal zur Erklärung der Rauhigkeit; man kann annehmen, dass eine Erhöhung des Dichteminimums um $6.5 \mathrm{~kg} / \mathrm{m}$ : eine Verbesserung der Rauhigkeit von ca. 40) p. 100 erbringt.

$$
\begin{gathered}
\text { Regu en mai } 1983 . \\
\text { Accepté en juin } 1983 .
\end{gathered}
$$

\section{Références bibliographiques}

Keititir R., Xivuxi: D. 1973. Méthode de la mesure des données microdensitométriques et de keur trationent à l'ordinateur. Communication présentée au Congres de l'I.U.F.R.O., Le' (al), Prétoriat. se'pt.oct.

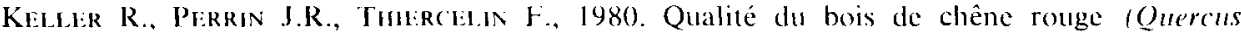
borealis Michaux) de quelques peuplements français. Document à distribution limitée, Station de Recherchess sur la Qwalité des Bois. C.N.R.F., 1980/1.

Kieleter R., Pierrin J.R., 1980. Rejations entre les résultats de laanaly'se densitométrique du bois de quelques résineux et la qualité des placages qu'ils sont susceptibles de produire. Docament à distribution limitée. Station de Recherches sur la Qualité des Bois, C.N.R.F., 1980/4.

Prrrin J.R., Firrani) J.C., 1984. Automatisation des mesures sur carottes de sondage de la densité du bois, de son retrait et des contraintes de croissance. Amm. Sci. Fom., 41 (1), $69-86$.

Porcal: H., 1964. Le bois juvénile des conifères. Rev. For. Fr., n" 6, 474-505.

POLGE H., 1966. Etablissement des courbes de variation de la densité du bois par exploration densitométrique de radiographies dechantillons prélevés à la tarière sar des arbres vivants. Applications dams les domaines technologique et physiologique. Thèse Doct. ès Sc. appl. Fac. Sc. Univ. Nancy I, 27 juin.

Thiercelin F., Perrin J.R., 1972. Débit d'échantillons d'épaisseur rigoureusement uniforme ì l'aide de scies-fraises jumelées. Ann. Sci. Ior., 30 (2), 295-299.

Tнов М., 1975. - Validité de tests précoces d'appréciation des caractères technologiques du bois de douglas. Application a une plantation comparative de 24 provenances de douglas (Peyrat-le-Château) en liaison avec des caractères morphologiques et atuxométriques. Rapport de stage E.N.I.T.E.F., Station de Recherchess sur la Qualité des Bois, C.N.R.F., juin. 